\title{
La educación física en la consecución de la competencia social y ciudadana Physical education in the achievement of social and citizen competence
}

\author{
Núria Monzonís Martínez, Marta CapllonchBujosa \\ Universitat de Barcelona
}

Resumen: Este artículo muestra el resultado de una investigación-acción, nacida de la necesidad de cambio del clima de convivencia de un centro de educación primaria de Barcelona. El propósito de la investigación fue dar respuesta a la hipótesis de acción: ¿Como puede contribuir el trabajo de la competencia social y ciudadana a la prevención y resolución pacífica de los conflictos desde el área de Educación Física? Para conseguirlo, una vez recogidos los datos del estado inicial del alumnado, se puso en práctica una estrategia de actuación para las sesiones de tutoría y de Educación Física, desarrollada por el grupo de trabajo participante en los ciclos de investigación-acción, y con objeto de favorecer la adquisición de la competencia social y ciudadana para fomentar la prevención y resolución pacífica de los conflictos. A lo largo de la aplicación se recogieron datos cualitativos y cuantitativos mediante cuestionario y observaciones participantes y sistemáticas. Los datos obtenidos reflejaron como resultados una mejora de los procesos relacionales. Hacia el final de la intervención las respuestas predominantes ante los conflictos fueron o bien ignorar el hecho, o bien el diálogo. En definitiva, se minimizaron las resoluciones que conllevaban violencia y aumentaron significativamente las mediaciones con ayuda de compañeros y el diálogo autónomo.

Palabras clave: Educación Física, competencia social y ciudadana, resolución de conflictos, Investigación-acción, convivencia, tutoría.

\begin{abstract}
This paper covers the results of an action research project stemming from the necessity of change in the climate of coexistence within a primary school in Barcelona. The investigation purpose was to give an answer to this action hypothesis: ¿How can the work of social and citizen competence contribute to the prevention and peaceful resolution of the conflicts from the physical education area? To achieve this, once the initial state of the students data was collected an action strategy for tutoring sessions and physical education was implemented. The strategy was developed by the teachers who participated in the work group with the aim to promote in the pupils the acquisition of skills of social and citizen competence to encourage prevention and peaceful conflict resolution. Qualitative and quantitative data were collected throughout the implementation using questionnaires, participant observation and systematic observation. Improvements of relational processes were shown in the obtained results. Towards the end of the intervention the predominant responses facing conflicts were either ignoring the fact or starting dialogue. As for the conflict resolution, those which entailed violence were minimized and on the other hand peer mediation and autonomous dialogue were significantly increased.
\end{abstract}

Key words: Physical Education, social and civic competence, conflict resolution, action research, coexistence, tutoring.

\section{Introducción}

Existe una creciente preocupación por el incremento de la violencia en los centros educativos motivado, entre otros, por las disfunciones sociales, los conflictos de convivencia, los altos niveles de agresividad, la fragilidad de la familias y el aumento de las conductas de riesgo durante la juventud, que han alertado sobre la necesidad de tratar este problema desde todos los niveles educativos (Marina \& Bernabeu, 2007).

Es bien cierto que la exposición a la violencia, en sus diferentes manifestaciones, constituye un potente factor a través del cual los niños y niñas adquieren patrones agresivos por imitación (Bandura, 1977; Coleman \& Fisher-Yoshida, 2004). En consecuencia, necesitamos proporcionar desde la escuela herramientas para neutralizar esta influencia y evitar que se comprometa su desarrollo emocional y social.

Por otro lado, en una sociedad cada vez más globalizada, plural y multicultural se hace evidente la necesidad de aprender a convivir y relacionarse con personas muy diferentes. Por ello será clave el desarrollo de habilidades sociales que permitan adaptarnos a un contexto como el actual. En consecuencia uno de los principales retos que deberá afrontar la educación será que las jóvenes generaciones adquieran, a lo largo de su proceso educativo, valores y actitudes necesarias tanto para el desarrollo social como personal (Velázquez \& Maldonado, 2004). GarcíaHierro y Cubo Delgado (2009), describen la convivencia escolar como aquellas relaciones socio-verbales que los integrantes del contexto educativo tienen entre sí, y que inciden significativamente en el desarrollo tanto ético, socio-afectivo como intelectual del alumnado.

Según Gázquez, Pérez y Carrión (2011) el clima escolar viene determinado por la relación existente entre los alumnos y los profesores en su quehacer diario, sin olvidar la importancia que tiene la participación e implicación de los padres en la escuela, no sólo para el propio

Fecha recepción: 05-12-13- Fecha envío revisores: 05-12-13- Fecha de aceptación: 10-12-13 Marta Capllonch Bujosa

Campus Mundet, Edifici Llevant, 3a planta, despatx 317,

Passeig del Vall d'Hebron 171, 08035 Barcelona,

mcapllonch@ub.edu desarrollo y desempeño académico del sujeto, sino también, en la mejora del clima de convivencia dentro de la escuela. Por lo tanto, un buen clima de convivencia favorecerá el correcto desarrollo de los procesos de enseñanza-aprendizaje y el desarrollo de personas con más valores de solidaridad, tolerancia y respeto (Selfridge, 2004). Cuando la convivencia se altera aparecen los conflictos nacidos de la contraposición de intereses, estos deben verse como un proceso natural necesario para el desarrollo psico-social y no solo como una amenaza.

Dada esta definición, podemos decir que los conflictos son inherentes a la naturaleza de las relaciones humanas y pueden originarse en cualquier proceso de convivencia. Por este motivo aprender a abordarlos debe ser un compromiso desde el ámbito educativo con objeto de minimizar las resoluciones negativas de estos y favorecer el desarrollo sociocognitivo de los estudiantes (Buchs, Butera, Mugny \& Darnpn, 2004; Coleman \& Fisher-Yoshida, 2004; Frydenberg, 2004; Pérez Fuentes, Gázquez, Fernández Baena \& Molero, 2011). Es importante afrontarlos positivamente en el momento y cuando surjan como si fueran un reto a conseguir por el alumnado (Frydenberg, 2004; Pérez de Guzmán, Amador \& Vargas, 2011).

Existen diversos enfoques para tratar el conflicto en el aula. Entre ellos, se destacan estrategias basadas en el diálogo y la convivencia(Díaz Aguado, 2005; Fernández García, 2008; García-Hierro García \& Cubo Delgado, 2009; Jares, 2006; Pérez Pérez, 2007), el trabajo cooperativo (Moliner Garcia \& Martí, 2002), el aprendizaje entre iguales (Ashby \& Neilsen-Hewett, 2012), la mediación (Johnson \& Johnson, 2004; Selfridge, 2004; Sellman, 2011), la autorregulación (Díaz Perea, 2005), etc. En nuestro caso, optamos por una perspectiva más globalizada que tenga en cuenta la adaptación del individuo al entorno mediante la adquisición de habilidades y competencias sociales.

Según Zabala y Arnau (2007), las competencias consisten en la intervención eficaz para una situación concreta, donde intervienen conocimientos, habilidades y actitudes simultáneamente. Blázquez (2009) añade que esos conocimientos, habilidades y actitudes pueden ser de diferentes saberes, por lo que será necesaria su comprensión, reflexión y discernimiento teniendo en cuenta la dimensión social de cada situación. 
La consecución de la competencia básica social y ciudadana (en adelante CSC) debe capacitar al alumnado en su realización personal, el ejercicio de la ciudadanía activa, la incorporación satisfactoria a la vida adulta y el desarrollo de un aprendizaje permanente a lo largo de la vida (Boluda \& Lecumberri, 2012; Méndez, López-Tellez \& Sierra, 2009). La Unión Europea define la CSC como los comportamientos que el individuo debe adquirir para participar constructivamente en la vida social, y resolver conflictos cuando sea necesario. Por otra parte, el Ministerio de Educación y Ciencia (2006), considera que es la competencia que permite comprender la realidad social en que se vive desde el ámbito cognitivo, que fomenta el cooperar, convivir y ejercer la ciudadanía democrática en una sociedad plural, desde el ámbito procedimental, y comprometerse a la mejora activa de la sociedad, desde el ámbito actitudinal. El conocimiento y comprensión de las sociedades democráticas, la práctica del diálogo y de la negociación son básicos para prevenir los conflictos y saber aportar soluciones cuando se producen. Por otro lado, saber expresar las propias ideas y escuchar las de los demás para tomar decisiones en función de los intereses del grupo, y el respeto, la cooperación y el compromiso con los valores necesarios para la convivencia, son ejes fundamentales de dicha competencia (González, Catalán \& Lara, 2011).

Una parte de esta competencia es fomentar la dimensión social de la persona para ir hacia una sociedad más justa y solidaria. Con ello fomentaremos que el alumnado comprenda y participe más activamente en la construcción de ideales y acciones del grupo social, y potenciaremos el desarrollo de capacidades y habilidades para relacionarse y comunicarse eficazmente (Boluda \& Lecumberri, 2012; Marina \& Bernabeu, 2007; Monjas, 2008; Segura \& Arcas, 2004; Zabala \& Arnau, 2007). Las habilidades sociales son las emociones, cogniciones y conductas necesarias para interactuar, relacionarse y convivir con los iguales y los adultos de forma efectiva y mutuamente satisfactoria (Monjas, 2008). Todo ello forma, según Goleman (2006), la inteligencia social formada por la conciencia social que permite percibir las emociones de los demás y la aptitud social que nos posibilita relacionarnos efectivamente.

La adquisición de la CSC será pues un elemento de mejora de la convivencia de vital interés para los centros educativos ya que, como defiende Goleman (2006), las personas están programadas para conectar con los demás y para las relaciones interpersonales.

La Ley Orgánica de Educación (2006), prescribe, mediante esta competencia, la educación en valores y en ciudadanía de manera transversal al currículo en todas las áreas y etapas. La Educación Física (en adelante EF) como área curricular no puede quedarse al margen de tales compromisos en la escuela, y habrá que ver pues qué contribución puede hacer el área a la CSC, y de qué manera, a través de ella se puede contribuir a la adquisición de habilidades para la prevención y resolución de conflictos que trasciendan a la vida escolar.

El carácter lúdico y vivencial del área hace que el alumnado se exprese espontáneamente y rompa con la rutina académica. Las acciones motrices conllevan una fuerte carga emocional donde las relaciones interpersonales y la comunicación serán constantes. Pese a estas características también se dan situaciones que propician los conflictos como discrepancias ante las acciones, por las diferencias entre niveles de habilidad o de la personalidad, por la cultura o el género, por el uso del material y del espacio, por la búsqueda excesiva de la victoria, etc. (Ortí, 2003). Estas situaciones convierten el área de EF en un entorno idóneo para el tratamiento del conflicto desde una perspectiva motivante para el alumnado. Existe un gran número de experiencias que han tratado de mejorar la convivencia desde el área de EF (Casey, Dyson \& Campbell, 2009; Castro \& González, 2006; Cecchini, Fernández, González \& Arruza, 2008; Cecchini, et al., 2009; Escartí, Pascual \& Gutiérrez, 2005; Gómez \& Prat, 2009; Gutiérrez, 2002; Hellison, 1995; Jiménez \& Durán, 2004; Mouratidou, Goutza \& Chatzopoulos, 2007; Pérez Pueyo, 2007; Prat \& Soler, 2003; Rodríguez Ruiz, 2010; Ruiz Omeñaca, 2004, 2008; Sánchez, Chinchilla, de Brugos \& Romero, 2008; C. Velázquez, 2007; R. Velázquez \& Maldonado, 2004; Venero, 2007). Sin embargo, existe un vacío en cuanto al trabajo concreto de la CSC desde la EF, a excepción de estudios conceptuales como es el caso de González, Catalán y Lara (2011); por eso desde esta investigación se pretende dar respuesta a la siguiente hipótesis de acción: ¿Como puede contribuir el trabajo de la CSC a la prevención y resolución pacífica de los conflictos en el área de EF? Dado este planteamiento nuestro objetivo principal será diseñar una línea estratégica de actuaciones basadas en el trabajo paralelo entre las sesiones de tutoría y el área de EF que favorezcan la adquisición de la CSC para fomentar la prevención y resolución pacífica de los conflictos, y promover relaciones positivas entre los miembros de los grupos.

Los objetivos específicos se centrarán en:

1. Identificar el número, causa y tipo de resolución de los conflictos que se dan durante las sesiones de EF

2. Crear un programa de intervención basado en la CSC para aplicar en las sesiones de tutoría y EF

3. Valorar la eficacia del programa a partir del análisis de las resoluciones de conflictos que se dan entre el alumnado

\section{Material y Método}

\section{Contextualización y participantes}

Este estudio fue llevado a cabo en un Instituto-Escuela público de Barcelona, situado en el distrito de Nou Barris. El nivel socio económico de las familias del centro es bajo y en algunas muy bajo debido al incremento en las tasas de desempleo. Aunque gran parte del alumnado es de origen inmigrante, también hay que señalar que se da un alto índice de familias de etnia gitana con dificultades de integración en la cultura escolar y con algunos problemas de cohesión. El centro nació en el curso 2011-12 de la fusión de dos centros de Educación Primaria del barrio y un Instituto de educación secundaria cercano. Tras el primer curso de adaptación los problemas de convivencia se llevaron al claustro de maestros, que decidió gestionar el problema, a partir de la propuesta de la profesora de EF y doctoranda de la Universidad de Barcelona.

La propuesta inicial se llevó a cabo a partir de una muestra participante formada por un total de 95 alumnos, con edades comprendidas entre 10 y 13 años, 56 niños con una edad media de 10.8 y 39 niñas con 10.9 de edad media. Estos estudiantes representaron cuatro grupos naturales, dos de quinto y dos de sexto de Educación Primaria. Para la selección de la muestra se siguió el criterio de accesibilidad, ya que eran los grupos en los que la investigadora impartía la EF, y el criterio de relevancia, porque eran las clases con más problemas de convivencia.

\section{Método}

La investigación está enmarcada dentro de la metodología cualitativa ya que queremos entender mejor los procesos relacionales que se dan entre el alumnado para tomar medidas que permitan mejorar la situación educativa. Dentro de esta metodología se desarrolla una actividad sistemática orientada a la comprensión en profundidad de fenómenos educativos y sociales, a la transformación de prácticas socioeducativas, a la toma de decisiones y al descubrimiento y desarrollo de un cuerpo organizado de conocimientos (Sandín, 2003).

Para conseguirlo seleccionamos la investigación-acción (I-A, en adelante) como método. Latorre, (2003) utiliza el término para describir el conjunto de actividades que utiliza el profesorado en sus propias aulas para: el desarrollo curricular, su autodesarrollo profesional, la mejora de los programas educativos, los sistemas de planificación o su política de aplicación. Todas estas actividades tienen en común que se planifican estrategias de actuación para aplicarse y ser sometidas a observación, reflexión y cambio a través de sus fases cíclicas (Latorre, 2004).

Este método nos permitió plantear un nuevo enfoque de aproximación a los conflictos en el centro y validar los cambios positivos de las nuevas estrategias pedagógicas (Casey, et al., 2009) a través de un seguimiento exhaustivo y su evaluación. Por otro lado, sus características se ajustaron a las necesidades de investigación, implicamos a los miembros de la comunidad educativa en el proceso y se consideraron agente de cambio para implementar el plan de acción y conseguir los 
propósitos establecidos (Latorre, 2004). Cambiar el clima escolar negativo es una tarea larga y difícil, que requerirá un esfuerzo de toda la escuela con una inversión considerable y el compromiso de los estudiantes, personal, administración y las familias (Gázquez, et al., 2011), por este motivo durante todo el ciclo existió un proceso de reflexión constante entre la propia práctica y la acción, los maestros recogieron los datos, colaboraron en la toma de decisiones y todos los participantes aprendieron progresivamente a partir de sus experiencias.

Fases de la I-A y técnicas de recogida de información

A lo largo de la I-A se siguieron las cuatro fases del ciclo de investigación propuestas por Kemmis y McTaggart (1992).

- Fase de planificación: Una vez identificado el problema, fruto de la reflexión del profesorado, en la fase de planificación se aplicaron dos instrumentos de recogida de datos para definir el estado inicial. Estos fueron un cuestionario, para el alumnado, diseñado a partir de las aportaciones de Capllonch (2007) y Ruiz Omeñaca (2008), y una observación sistemática de las sesiones de EF del tercer trimestre del curso 2011-12. El cuestionario tenía como objetivo captar la percepción del alumnado sobre el clima de convivencia en el centro, por lo que se dividió en dos grandes dimensiones: el ámbito de aula y el de EF. Las cuestiones planteadas para la parte específica giraban en torno a la percepción del clima de convivencia en las sesiones de EF; la percepción del alumnado sobre su comportamiento, las causas que provocaban los conflictos o hacían que no funcionaran las actividades, las reacciones ante los problemas, los modelos de resolución de los conflictos que se daban y por los que ellos optarían.

La observación sistemática nos sirvió para detectar el número, causa y tipo de resolución de los conflictos que se daban en los grupos de ciclo superior. Ésta se llevó a cabo con la ayuda de un observador entrenado que analizó 18 sesiones de EF del tercer trimestre del curso 2011-12.

Los datos obtenidos permitieron redactar el informe de diagnóstico con el que se fijaron las necesidades del centro y se creó un grupo de trabajo formado por siete maestros voluntarios de educación infantil, educación primaria y educación secundaria. Durante el último trimestre del curso 2011-12 y el primero del 2012-13, el grupo diseñó el plan estratégico para los tutores de quinto y sexto, y para la maestra de EF y además planteó las técnicas de recogida de datos para la fase de observación. El programa de intervención basado en la interrelación entre la sesión de tutoría y la de EF, se elaboró a través de diferentes referencias (Boqué \& Torremorell, 2002; Escartí, et al., 2005; Hellison, 1995; Monjas, 2008; Pérez Pueyo, 2007; Prat \& Soler, 2003; Ruiz Omeñaca, 2008; Segura \& Arcas, 2004) adaptadas a las características de la escuela.

- Fase de acción: La fase de acción se desarrollo durante el curso 2012-13 aplicando el programa en una sesión semanal de una hora de tutoría y dos sesiones, de una hora también, de EF. En total se realizaron 26 sesiones de tutoría y 54 de EF. En los dos grupos de quinto se siguió rigurosamente el programa y en los de sexto sólo se aplicó la parte correspondiente a EF debido a cambios entre el profesorado de ese nivel.

Para implementar el programa se aplicaron herramientas de reflexión y transformación como: razonamiento moral a través de historias hipotéticas que contraponen valores, debates breves de concienciación de los valores tratados, elaboración de carteles resumen de los acuerdos consensuados democráticamente por el alumnado, aprendizaje entre iguales, autoevaluación de cada sesión y respeto del ritmo de aprendizaje (Cecchini, Montero \& Peña, 2003; Jiménez \& Durán, 2004).

Las sesiones de EF siguieron la estructura del modelo de Hellison (1995): presentación inicial de los objetivos en valores o conductas a seguir en clase, actividad con momentos de reflexión si es necesario y finalización con diez minutos de evaluación, autoevaluación y reflexión sobre las dinámicas dadas en clase.

- Fase de observación: Durante la fase de observación, paralela a la acción, controlamos y registramos el plan de acción con la intención de generar datos para la reflexión y evaluación. Gracias a ello, pudimos

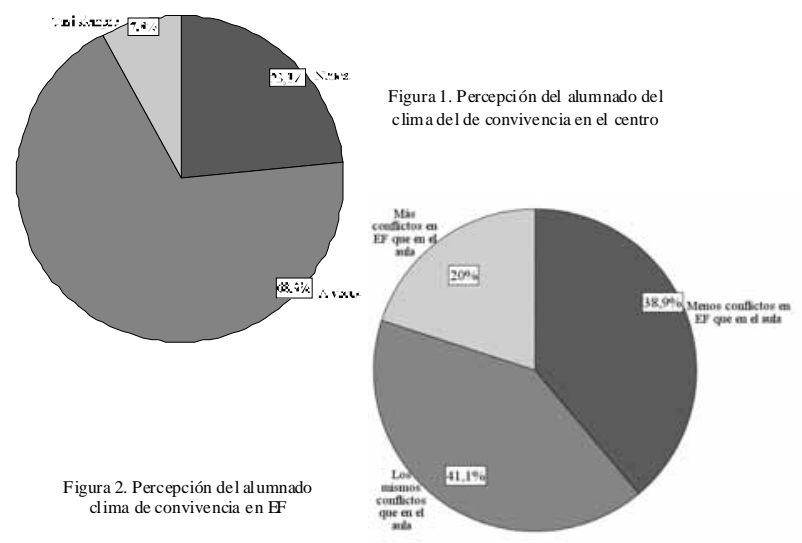

detectar y mejorar ciertas debilidades de la planificación inicial y adaptarnos a los contratiempos del curso escolar. A lo largo de todo el proceso la maestra de EF aplicó la observación participante, como técnica inherente a la I-A que facilita llegar a las personas clave de la investigación sin modificar su comportamiento (Latorre, 2003). La docente recopiló en un diario las actitudes y relaciones que se dieron entre el alumnado y pudo captar la complejidad de las situaciones, interpretarlas y reflexionar sobre ellas. El diario planteado era abierto, por lo que también se incluyeron descripciones y narraciones de los hechos observados lo más exactos y completos posibles que llevaron a una mejor comprensión de las interacciones de los alumnos (Gómez, Latorre, Sánchez \& Flecha, 2006).

Hacia el final de la acción se recurrió de nuevo a la observación sistemática de 22 sesiones de EF del tercer trimestre del curso 2012-13, con la colaboración de un observador entrenado. Esta observación nos sirvió para comparar los resultados de manera directa con los del inicio del proceso, ya que en ella se tuvieron en cuenta las mismas categorías pre-establecidas a través de las aportaciones de Ruiz Omeñaca (2008).

- Fase de reflexión: Por último, en la fase de reflexión se llevó a cabo la tarea de análisis que nos permitió extraer evidencias de las consecuencias del plan de acción y detectar sus puntos débiles. Estas tres herramientas (cuestionario, observación sistemática, observación participante) posibilitaron triangular y dar solidez a los resultados obtenidos a través de los datos que reflejaban los efectos relevantes y dar respuesta a la hipótesis de acción.

\section{Resultados}

El cuestionario para el alumnado junto con las observaciones sistemáticas iniciales y finales se analizaron a través del software PASW Stadistics 18 (SSPS) y los datos cualitativos, obtenidos del diario de la maestra de EF, se estudiaron con el software Nvivo 1.1., aplicando un proceso deductivo e inductivo. El punto de partida deductivo fueron tres categorías: causas del conflicto, respuestas que se daban y el modo

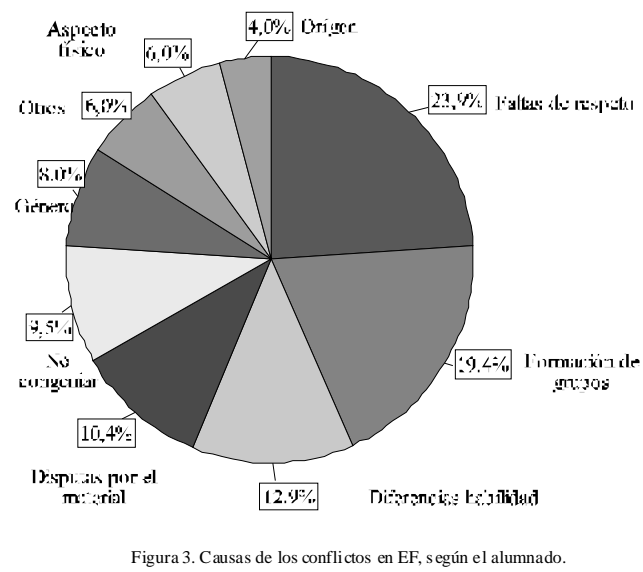




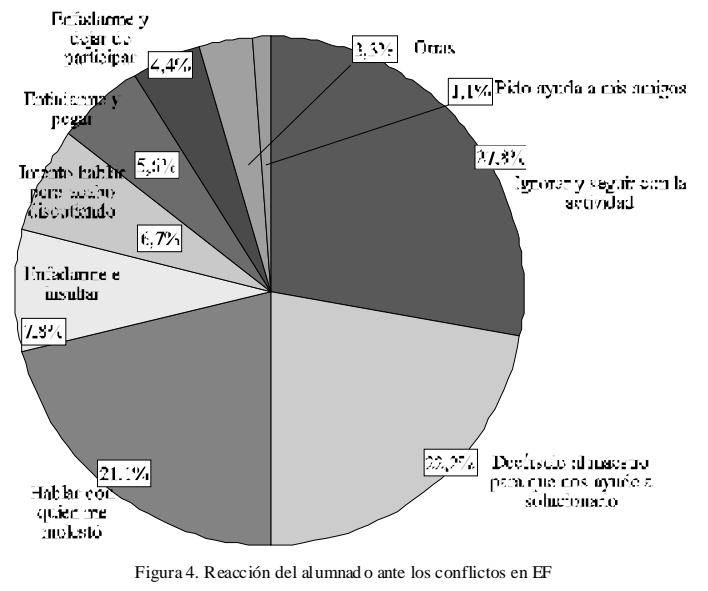

de resolución, pero a lo largo del análisis de datos se obtuvieron nuevas categorías y subcategorías inductivas. Entre las creadas a posteriori debemos destacar el tipo de actividad, que nos permitió detectar en que situaciones se producían más conflictos, y las conductas pro-sociales, que se daban entre el alumnado: ayudas mutuas, preocuparse por la participación y bienestar de los demás, empatía o cooperación.

Los datos del cuestionario inicial sirvieron de punto de partida para valorar la percepción de alumnado sobre el clima de convivencia tanto del centro escolar, como de las sesiones de EF. Los datos revelaron que un 68.9\% de los niños y niñas de ciclo superior de Educación Primaria se sentía a veces mal en clase por el comportamiento de sus compañeros. Sin embargo, la percepción fue que en las clases de EF se daban igual o menor número de conflictos que en el aula. Las figuras 1 y 2 muestran la comparativa de las percepciones del alumnado sobre el clima de la clase en el aula y en EF.

Según las respuestas del alumnado las causas que originan conflictos en el área de EF son las faltas de respeto (23.8\%), los problemas que se generan en la formación de grupos (19.4\%) y las derivadas por los diferentes niveles de habilidad (12.9\%). Otras causas mencionadas son: las disputas por el material (10.4\%); diferencias de la personalidad (9.4\%) y los conflictos por razón de género (7.9\%). Las categorías con menores porcentajes son las producidas por el aspecto físico (5.9\%) y el origen (3.9\%). A la luz de estos resultados podemos afirmar que la multiculturalidad del centro, y el bajo nivel económico de las familias no son la causa del conflicto, sino que ésta viene provocada en la mayoría de las situaciones por la falta de respeto hacia los demás. La Figura 3 muestra la relación de comportamientos mencionados por el alumnado que generan conflictos en la clase de EF.

A la cuestión planteada sobre lo que creen los alumnos que hace que no funcionen las actividades de EF, el $41.1 \%$ menciona que son las trampas, el 22\% la competitividad y el 20\% la agresividad de los compañeros. En un segundo término destacan la escasa participación de algunas personas en las actividades (7.8\%), o bien el hecho de que algunos alumnos no dejen participar a sus compañeros (4.4\%).

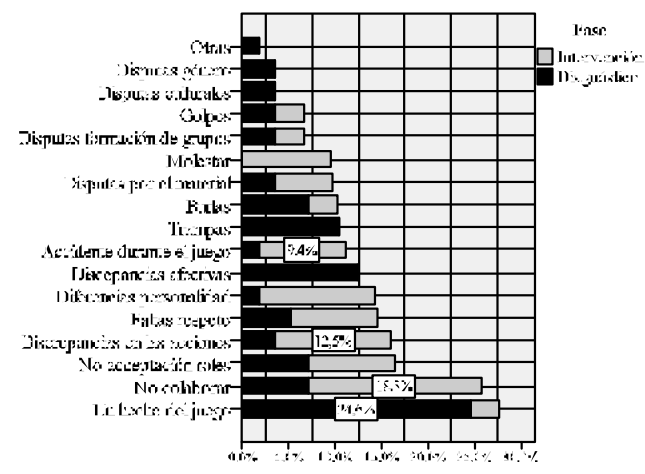

Figura 5. Causas de los conflictos antes y durante la intervención

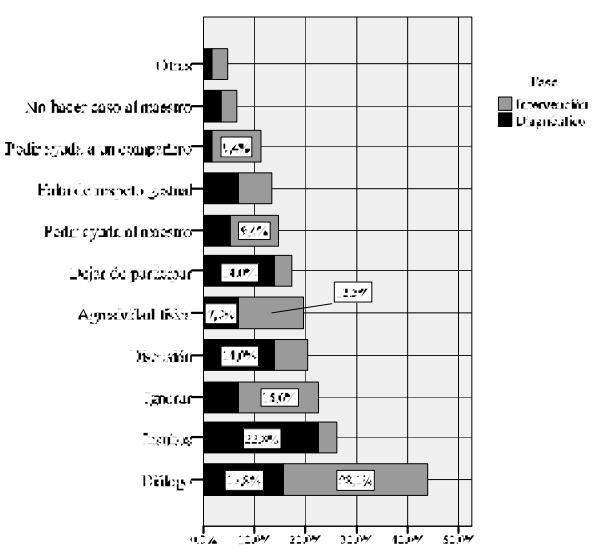

Figura 6. Respuestas inmediat as ante los conflict os antes y durante la intervención

Las reacciones frente al conflicto presentan tres patrones mayoritarios que son: ignorar el hecho conflictivo y seguir jugando (27.7\%), decírselo al maestro para que lo solucione o colabore en su resolución (22.2\%) o bien intentar hablar con la persona causante del conflicto (21.1\%). Las otras reacciones con menor trascendencia son: contestar enfadado e insultar (7.7\%); discutir con la otra persona (6.6\%), pegar (5.5\%), dejar de participar en la actividad (5.5\%) y pedir ayuda a los compañeros (1.1\%). La figura 4 ilustra dichos resultados.

En referencia al modelo de resolución de conflictos que el alumnado menciona que llevaría a cabo, el 34.4\% propone que sea el maestro el que hable con los implicados, y si fuera necesario que aplique un castigo. El 33.3\% considera que sería mejor hablar de la situación en clase y buscar una solución entre todos y todas. Un $11.1 \%$ del alumnado optaría porque una persona ajena al conflicto hiciera de mediadora. También un $11.1 \%$ optaría directamente por un castigo. Los resultados muestran que el alumnado reproduce los pasos que el centro sigue habitualmente frente a los conflictos: hablar con las personas afectadas, y aplicar castigos en caso de la situación sea de importancia.

Dadas estas evidencias en el grupo de trabajo de I-A nos planteamos conseguir mejorar el clima de convivencia en el aula, mejorar las estrategias de trabajo cooperativo para minimizar los conflictos negativos, trabajar la educación emocional para lograr un mejor equilibrio de sus reacciones afectivas, el cumplimiento de las normas como medio de mejora de la convivencia, y favorecer la resolución autónoma y pacífica de los conflictos por parte del alumnado huyendo de actitudes negativas y minimizando la actuación del profesorado.

Una vez aplicado el programa de intervención obtuvimos resultados significativos que evidencian un cambio en las dinámicas de los grupos. Mientras que en la fase de planificación la mayoría de conflictos estaban vinculados a hechos del juego (24.6\%), durante la intervención la tendencia se desvió hacia situaciones más genéricas del trabajo cooperativo como las discrepancias en las acciones (12.5\%) o no colaborar en las actividades (18.8\%), como así lo demuestran algunos párrafos extraídos del diario de la maestra:

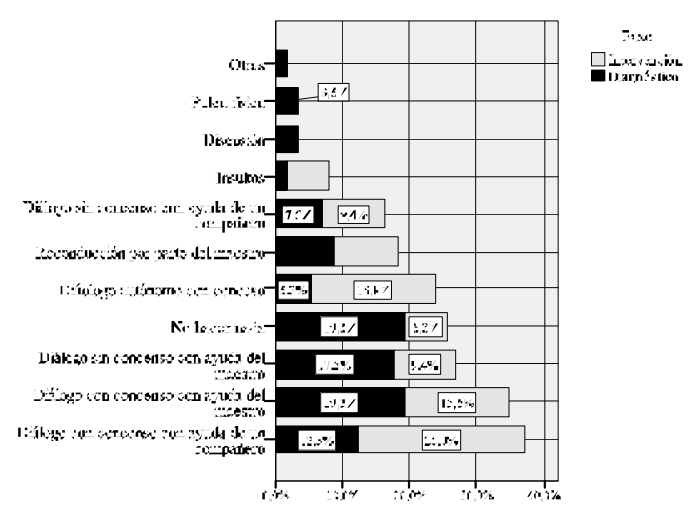


Una vez terminamos la unidad didáctica de gimnasia cooperativa evaluamos como se corrigen entre ellos lo elementos técnicos tratados. Hay dos grupos que tienen la dinámica de grupo asimilada y han hecho grandes avances, pero el grupo de Serafín, Rubén,Alberto y Vicente no trabajan demasiado no colaboran entre ellos y están jugando todo el rato a tirarse uno encima del otro y siempre acaban discutiendo porque no consiguen hacer las actividades propuestas.

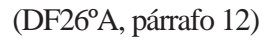

La figura 5 muestra las diferencias de las causas de los conflictos antes de la intervención y durante la misma.

En cuanto a las respuestas inmediatas frente a los conflictos también se ha detectado un cambio substancial como podemos ver en la figura 6. Mientras que al inicio del proceso los insultos (22.8\%), el dejar de participar (14\%) y las discusiones (14\%) fueron mayoritarios; en el tercer trimestre del curso las respuestas predominantes han sido ignorar el hecho (24.5\%) y el diálogo (24.5\%) y en segundo término el pedir ayuda a compañeros (9.4\%) o maestro (9.4\%).

A continuación se presentan algunas muestras del diario del docente que evidencian casos específicos de este cambio favorable.

Emilio chilla a Britany porque no entiende como se lleva a cabo la actividad, Yanira interviene y le pide que se lo diga bien y se lo explique con tranquilidad, Emilio se da cuenta que se ha equivocado y le pide perdón y se lo explica bien (DF25ํㅡ párrafo 36)

En referencia a la última variable destacada, la resolución de los conflictos también se ha dado una tendencia favorable en las resoluciones positivas. En la fase de diagnóstico existía un 3.5\% de peleas físicas y durante la intervención no se dio ninguna resolución a través de la violencia. Por otro lado han aumentado un $6 \%$ las mediaciones con ayuda de un compañero y un $11 \%$ las resoluciones a través del diálogo de manera autónoma. Por otro lado, cabe destacar que dejar pasar el conflicto sin hacer nada también ha disminuido en un $7 \%$. En las figura 7 se puede observar las estrategias de resolución de conflictos antes y durante la intervención. Asimismo el siguiente fragmento nos ofrece indicios de dicha transformación.

Hoy durante el juego libre se ha dado una situación un poco tensa, mientras jugaban a futbol, Federico y Maiquel se han puesto nerviosos porque estaban perdiendo, comentaban que Alex tenia la culpa y que tendrían problemas. Después ha intervenido David y les ha pedido que se calmaran, les ha dicho que seguro que si no perdieran no estarían nerviosos, ellos han dicho que tal vez tiene razón y con tranquilidad le han dicho aAlex que les parecía queél les provocaba, este lo ha negado e incluso les ha pedido perdón por si había hecho algo que les molestara. Esta resolución era inimaginable a principio de curso, Maiquel y Federico son dos niños nerviosos que antes siempre recurrían al insulto, la amenaza y el golpe para solucionar las cosas y David siempre les acompañaba. La actitud de David ha cambiado totalmente, antes en una situación así no hubiera puesto paz, hubiera animado a los compañeros para que se peleasen. (DF35 A párrafo 63) ${ }^{1}$

Los resultados sugieren un impacto positivo del programa ya que las resoluciones que derivan en acciones violentas se han minimizado, los alumnos han mejorado sus habilidades comunicativas y emocionales y son capaces de recurrir al diálogo o la demanda de ayuda externa para solucionar sus problemas. Además el trabajo cooperativo y aprendizaje entre iguales ha dado sus frutos ya que los cuatro grupos son capaces de sacar adelante proyectos en equipo. El trabajo en parejas o pequeños grupos les ayudó a ser capaces de ofrecer orientaciones a sus compañeros, a aprender a escuchar y esperar su turno de palabra y a resolver problemas a través del diálogo y el consenso.

El progreso de los alumnos para trabajar en equipo ha sido espectacular, los niños y niñas de las cuatro clases han sido capaces de crear pequeñas coreografías. En todos los grupos se han implicado y han aportado ideas, todos han disfrutado del proceso y están satisfechos con los resultados. En algunos grupos había alumnos que dirigían más al grupo, pero no ha sido un problema ya que los demás confiaban en su criterio y también colaboraban (DF3, párrafo 68)
Para un siguiente ciclo se podrían plantear diversas opciones: desde ampliar la actuación al resto de grupos del centro, aumentar la transversalidad del proyecto, incrementar la participación de las familias o incluso centrarnos en establecer un servicio de mediación dirigido por el alumnado.

\section{Discusión y conclusiones}

La escuela debe ofrecer a los alumnos la adquisición de las habilidades y destrezas para afrontar los retos de la vida diaria (Frydenberg, 2004; Zabala \& Arnau, 2007). El área de EF contribuye de forma esencial al desarrollo de la CSC ayudando a aprender a convivir, desde la elaboración y aceptación de las reglas, hasta el respeto a la autonomía personal, la participación y la valoración de la diversidad (Blázquez, 2009; González, et al., 2011; Méndez, et al., 2009). Las actividades dirigidas a la adquisición de las habilidades motrices requieren la capacidad de asumir las diferencias así como las posibilidades y limitaciones propias y ajenas. El cumplimiento de la norma en el juego colabora en la aceptación de códigos de conducta para la convivencia. Las actividades físicas competitivas pueden generar conflictos en los que es necesaria la negociación, basada en el diálogo, como medio de resolución (Ciencia, 2006; Chinchilla, Escribano, Romero \& López, 2008; Gómez \& Prat, 2009).

Estas características del área de EF se han aprovechado para centrarnos en la mejora de un aspecto concreto de la CSC, la resolución de conflictos. Con ello queremos afirmar que a lo largo de todo el programa de intervención estas habilidades siempre han estado presentes y han sido un soporte esencial para lograr dichos avances. Los alumnos y alumnas necesitan experimentar que el conflicto se puede solucionar pacíficamente, por lo que la escuela ha creado un entorno en el que, a diario, se vivan relaciones de cooperación y resoluciones positivas de los problemas entre los compañeros (Adrià Casans, Garcia Peruga, Mateu Miralles, Pérez Fernández \& Sànchez Alcón, 2012; Álvarez García, et al., 2009; Ashby \& Neilsen-Hewett, 2012; Escartí, et al., 2005). El trabajo cooperativo y el aprendizaje entre iguales ha permitido que aparezcan interrelaciones sociales y situaciones controvertidas donde el alumnado debe ser capaz de aplicar sus habilidades comunicativas, emocionales y sociales para complementar sus puntos de vista con los del compañero, y así llegar a soluciones creativas de las tareas (Buchs, et al., 2004; Fernández Río, 2003; López, 2009; Velázquez, 2007). Mejorando las habilidades sociales se mejorarán los intercambios y la calidad de las interacciones del trabajo en grupo, y ello también influirá en el incremento de los aprendizajes académicos (Johnson \& Johnson, 2004).

Según Selfridge (2004), el nivel de logros del programa esta directamente relacionado con el nivel de compromiso del personal docente, por ello creemos que los resultados hubieran sido aún más positivos en todos los grupos si se hubiera contado con un mayor soporte de todo el profesorado, ya que, aunque no era lo esperado, solo las tutoras de quinto fueron las que acabaron siendo el motor del proceso.

Valoramos que la combinación del programa en las sesiones de tutoría y EF ha fortalecido la acción y ha permitido crear un buen clima de convivencia y favorecer la autonomía de los alumnos para dialogar ante los conflictos. En el aula se trataban las habilidades a través de planteamientos hipotéticos y en la pista o el gimnasio se encontraban inmersos en situaciones reales donde debían aplicar lo debatido en clase para poder lograr los objetivos programados. Consideramos que dicha transferencia ha sido la clave del éxito para el alumnado y el elemento que nos ha dado firmeza para impulsar un nuevo ciclo de I-A.

\section{Referencias}

Adrià Casans, M.P., Garcia Peruga, M., Mateu Miralles, P., Pérez Fernández, M.A., \& SànchezAlcón, C. (2012). Dialogue, per tant, convisc. Propostes didàctiques per a resoldre conflictes de manera crítica i creativa. Guix, 388 77-81.

Álvarez García, D., Álvarez Pérez, L., Núñez Pérez, J.C., Rodríguez Pérez, C., González Pienda, J.A., \& González Castro, P. (2009). Efectos sobre la conflictividad escolar de un programa de educación en resolución de conflictos en tutoría. International Journal of Psychology and Psychological Therapy, 9 (2) 189-204.

Ashby, N., \& Neilsen-Hewett, C. (2012). Approaches to Conflict and Conflict 
Resolution in Toddler Relationships. Journal of Early Childhood Research, 10(2), 145-161.

Bandura, A. (1977). Self-efficacy: toward a unifying theory of behavioral change. Physchological Review, 84, 197-215.

Blázquez, D. (2009). Enseñar por competencias en Educación Física. Barcelona: Inde.

Boluda, G., \& Lecumberri, C. (2012). Concepte, dimensions i justificació de les competències. Guix, 382, 13-16.

Boqué i Torremorell, M.C. (2002). Guia de mediació escolar. Programa comprensiu d'activitats. Educació primària i secundària obligatòria. Barcelona: Rosa Sensat.

Buchs, C., Butera, F., Mugny, G., \& Darnpn, C. (2004). Conflict Elaboration and Cognitive Outcomes. Theory Into Practice, 43(1), 23-30.

Capllonch, M. (2007). Informe 1. Teorías i prácticas sobre prevención y resolución de conflictos a través de la E.F. Plan Nacional I+D 2007-2009. Juega, dialoga y resuelve: la superación de conflictos en E.F. mediante el modelo comunitario. Diseño de un programa especifico para Comunidades de aprendizaje. Universidad de Barcelona. Centro especial de investigación en teorías y prácticas superadoras de desigualdades. Barcelona.

Casey, A., Dyson, B., \& Campbell, A. (2009). Action research in physical education: focusing beyond myself through cooperative learning. Educational Action Research, 17(3), 407-423.

Castro, M.J., \& González, M.A. (2006). Actitudes y motivación en Educación Física escolar. Retos. Nuevas tendencias en Educación Física, Deporte y Recreación., 10, 5-22.

Cecchini, J.A., Fernández, J.L., González, C., \& Arruza, J. (2008). Repercusiones del Programa Delfos de educación en valores a través del deporte en jóvenes escolares. Revista de Educación, 346, 167-186.

Cecchini, J. A., González, C., Alonso, C., Barreal, J. M., Fernández, C., García, M., . . \& \& Nuño, P. (2009). Repercussions del Programa Delfos sobre els nivells d'agressivitat en l'esport i en altres contexts de la vida diària. Revista Apunts EF i Esports, 96, 34-41.

Cecchini, J.A., Montero, J., \& Peña, V. (2003). Repercusiones del Programa de Intervención para desarrollar la Responsabilidad Personal y Social sobre los comportamientos de fair-play y el auto-control. Psicothema, 15, 631-637.

Ley Orgánica 2/2006, de 3 de mayo (2006).

Coleman, P., \& Fisher-Yoshida, B. (2004). Conflict Resolution at Multiple Levels Across the Lifespan. The work of the ICCCR. Theory Into Practice, 43(1), 31 38.

Chinchilla, J.L., Escribano, M.F., Romero, O., \& López, I. (2008). Análisis de las Actitudes de la Educación Física en la E.S.O. Retos. Nuevas tendencias en Educación Física, Deporte y Recreación, 14, 70-74.

Díaz Aguado, M.J. (2005). Educar para la tolerancia y prevenir la violencia un año después del 11-M. Cuadernos de Pedagogía, 344, 54-58.

Díaz Perea, M. R. (2005). La resolución de conflictos en el aula como parte de proceso de autonomía. Papeles de trabajo sobre Cultua, Educación y Desarrollo Humano, 1(2). Recuperado en: http://www.uam.es/otros/ptcedh/ 2005v1_pdf/v1n2esp.pdf

Escartí, A., Pascual, C., \& Gutiérrez, M. (2005). Responsabilidad personal y social a través de la educación física y el deporte. Barcelona: Graó.

Fernández García, I. (2008). Los programas de ayuda para la mejora de la convivencia en instituciones educativas. Bordón, 60(4), 135-150.

Fernández Río, J. (2003). El aprendizaje cooperativo en el aula de educación física para la integración en el medio social.Análisis comparativo con otros sistemas de enseñanza y aprendizaje. Valladolid: La Peonza.

Frydenberg, E. (2004). Coping Competencies: What to teach and When. Theory Into Practice, 43(1), 14-22.

García-Hierro García, M.A., \& Cubo Delgado, S. (2009). Convivencia escolar en Secundaria: aplicación de un modelo de mejora del clima social. Reviste Electrónica Interuniversitaria de Formación del Profesorado, 12(1), 51-62.

Gázquez, J.J., Pérez, M.C., \& Carrión, J.J. (2011). Clima escolar y resolución de conflictos según el alumnado: un estudio europeo. Revista de Psicodidáctica 16(1), 39-58.

Goleman, D. (2006). La inteligencia social. Barcelona: Kairós.

Gómez, I., \& Prat, M. (2009). Hacia una Educación Física y transformadora de las prácticas sociales: de la práctica a la refelxión y viceversa. Cultura y Educación, 21(1), 9-17.

Gómez, J., Latorre,A., Sánchez, M., \& Flecha, R. (2006). Metodología comunicativa crítica. Barcelona: El Roure.

González, S., Catalán, M., \& Lara, J.M. (2011). La competencia social y ciudadana en Educación Física. In O. R. Contreras \& R. Cuevas (Eds.), Las competencias básicas desde Educación Física (pp. 63-77). Barcelona: INDE.

Gutiérrez, J. (2002). Reflexiones sobre el papel de la actividad física y el deporte en edad escolar en la promoción de valores. Retos. Nuevas tendencias en Educación Física, Deporte y Recreación, 2002, 15-20.

Hellison, D.R. (1995). Teaching personal and social responsability through physical activity. Champaign: Human Kinetics.

Jares, X.R. (2006). Pedagogía de la convivencia. Barcelona: Graó.

Jiménez, P.J., \& Durán, L.J. (2004). Propuesta de un programa para educar en valores a través de la actividad física y el deporte. Apunts, 77, 25-29.

Johnson, D.W., \& Johnson, R.T. (2004). Implementing the «Teaching Students to be Peacemakers Program». Theory Into Practice, 42(2004), 68-79. doi:10.1207/ s15430421tip4301_9

Kemmis, S., \& McTaggart, R. (1992). Cómo planificar la Investigación-Acción. Barcelona: Laertes.

Latorre, A. (2003). La investigación-acción. Conocer y cambiar la práctica educativa. Barcelona: Graó.

Latorre, A. (2004). La investigación acción. In R. Bisquerra (Ed.), Metodologia de la investigación cualitativa. (pp. 369-394). Madrid: La Muralla.

López, V.M. (2009). El lugar de las actividades físicas cooperativas en una programación de Educación Física por dominios de acción. Retos. Nuevas tendencias en Educación Física, Deporte y Recreación, 16, 36-40.

Marina, J.A., \& Bernabeu, R. (2007). Competencia social y ciudadana. Madrid: Alianza.

Méndez, A., López-Tellez, G., \& Sierra, B. (2009). Competencias Básicas: sobre la exclusión de la comptetencia motriz y las aportaciones desde la Educación Física. Retos. Nuevas tendencias en Educación Física, Deporte y Recreación, 16, 51-57.

Moliner Garcia, O., \& Martí, M. (2002). Estrategias didácticas para la solución cooperativa de conflictos y toma de decisiones consensuadas: mejorar la convivencia en el aula. Revista Electrónica Interuniversitaria de Formación del Profesorado,5(3). Recuperado en: http://www.aufop.com/aufop/uploaded files/ articulos/1227713324.pdf

Monjas, M.I. (2008). Como promover las convivencia. Programa de asertividad y habilidades sociales. (PAHS). Madrid: Cepe.

Mouratidou, K., Goutza, S., \& Chatzopoulos, D. (2007). Physical education and moral development: An intervention programme to promote moral reasoning through physical education in high schools students. European Physical Education Review, 13(1), 41-56.

Ortí, J. (2003). La resolución de conflictos en la Educación Física. Tándem. Didáctica de la Educación Física, 13, 40-50.

Pérez de Guzmán, V., Amador, L., \& Vargas, M. (2011). Resolución de conflictos en las aulas: un análisis desde la Investigación-Acción. Pedagogía Social. Revista Interuniversitaria, 18(1139-1723), 99-104.

Pérez Fuentes, M.C., Gázquez, J.J., Fernández Baena, R., \& Molero, M.M. (2011) Análisi de las publicaciones sobre convivencia escolar en una muestra de revistas de educación en la última década. Aula Abierta, 39(2), 81-90.

Pérez Pérez, C. (2007). Efectos de la aplicación de un programa de educación para la convivencia sobre el clima social del aula en un curso de $2^{\circ}$ de E.S.O. Revista de Educación, 343, 503-529.

Pérez Pueyo,A. (2007). La organización secuencial hacia las actitudes: Una experencia sobre la intencionalidad de las decisiones del profesorado de educación física. Tándem didáctica para la Educación Física, 25, 81-92.

Prat, M., \& Soler, S. (2003). Actitudes, valores y normas en EF y el deporte. Barcelona: Inde.

Rodríguez Ruiz, D. (2010). El fútbol como herramienta para el trabajo de los valores y las actitudes en la ESO según las Competencias Básicas. Retos. Nuevas tendencias en Educación Física, Deporte y Recreación, 17, 67-71.

Ruiz Omeñaca, J.V. (2004). Pedagogía de los valores en EF. Como promover el pensamiento ético y la sensibilidad moral desde las actividades físicas y deportivas.: Consejo Superior de Deportes.

Ruiz Omeñaca, J.V. (2008). Educación Física, valores éticos y resolución de conflictos: reflexiones y popuestas de acción. In A. Fraile (Ed.), La resolución de conflictos en y a través de la educación física. (pp. 65-116). Barcelona: Graó. Biblioteca Tándem.

Sánchez, J.F., Chinchilla, J.L., de Brugos, M., \& Romero, O. (2008). Las relaciones sociales y educativas existentes entre los elementos personales del proceso educativo durante una sesión de Educación Física. Un estudio de casos. Retos. Nuevas tendencias en Educación Física, Deporte y Recreación, 14, 66-69.

Sandín, M.P. (2003). La investigación cualitativa en Educación. Fundamentos y tradiciones. Madrid: Mc Graw-Hill.

Segura, M., \& Arcas, M. (2004). Relacionarnos bien: programa de competencia social para niños y niñas de 4 a 12 años. Madrid: Narcea.

Selfridge, J. (2004). The Resolving Conflict Creatively Program: How We Know It Works. Theory Into Practice, 43(1), 59-67.

Sellman, E. (2011). Peer Mediation Services for Conflict Resolution in Schools: What Transformations in Activity Characterise Successful Implementation? British Educational Research Journal, 37(1), 45-60.

Velázquez, C. (2007). Educación Física para la paz: aprendizaje cooperativo. Novedades Educativas(198), 12-15.

Velázquez, R., \& Maldonado, A. (2004). Las actitudes y su evaluación en E.F. In L. Hernández \& R. Velázquez (Eds.), La evaluación en E.F. Investigación y práctica en el ámbito escolar. (pp. 169-202). Barcelona: Graó.

Venero, J.P. (2007). La clase de Educación Física como motor de cambio social. Reflexionando sobre actividades en la naturaleza, curriculum oculto y valores. Retos. Nuevas tendencias en Educación Física, Deporte y Recreación, 11, 5153.

Zabala, A., \& Arnau, L. (2007). 11 ideas clave. Cómo aprender y enseñar competencias. Barcelona: Graó.

Notas al pie

${ }^{1}$ En los fragmentos del diario se han modificado los nombres de los alumnos para mantener su anonimato. 